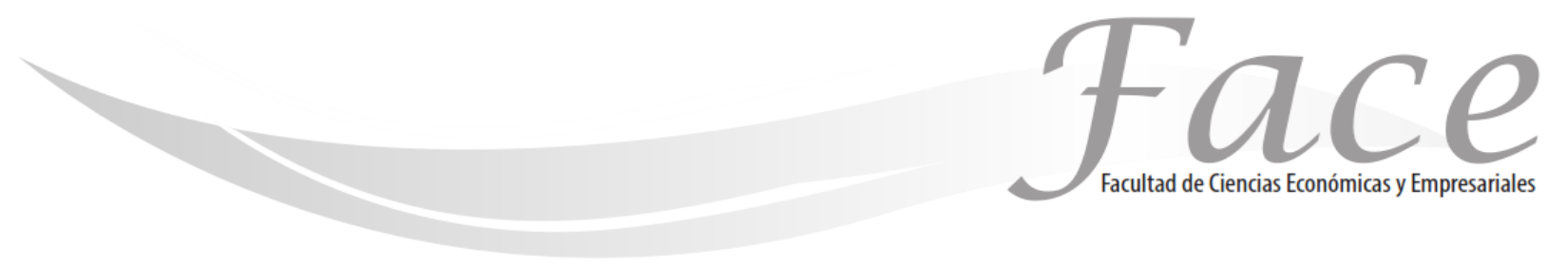

ISSN 1794-9920

Volumen $15-\mathrm{N}^{\circ} 2$

Año 2015

Págs. 38 - 51

\title{
LA PERCEPCIÓN DE LA MERITOCRACIA EN EL PROCESO DE SELECCIÓN DE PERSONAL EN LAS EMPRESAS COLOMBIANAS
}

\author{
Mónica Alejandra Pastrana Granados * \\ Franklin Ariel Prieto Pinto **
}

Fecha de Recepción: 2 de Agosto 2015

Fecha de Aprobación: 4 de Noviembre 2015

\section{Resumen:}

El concepto de meritocracia, hoy en día se puede relacionar con los tipos de sociedades y formas de gobierno de un país, ya que para muchas personas para que exista la meritocracia debe prevalecer la democracia en un sistema político, por lo contario se denominaría dictadura y es por esta razón que se consideró necesario investigar cómo se encuentra el estado actual de la selección y valoración del personal basado en la meritocracia de las empresas colombianas. Con el presente documento se muestran los resultados de una investigación tipo descriptivo, aplicada a empleados de todos los niveles organizacionales, incluyendo pero no exclusivamente a cargos directivos estratégicos y de nivel táctico de empresas ubicadas en la zona geográfica de Bogotá D.C.

El instrumento de recolección empleado para la respectiva investigación fue una encuesta que constaba de preguntas tipo cerradas, cuyo objetivo principal era el de conocer la percepción de los empleados respecto a los criterios de evaluación meritocráticos en el proceso de selección y valoración del personal. Los resultados generales de la investigación, proyecta que las variables que prevalecen en el proceso de selección del personal en la mayor parte de las empresas de Bogotá D.C, está determinado por referencias personales, experiencia laboral y nivel de estudios académicos, que son pocas las empresas que tienen un manual de reclutamiento y selección de personal, sino que en la mayoría de ocasiones la decisión final del puesto vacante está determinada por la persona quién administra el cargo de jefe de Desarrollo Humano o gerente de la empresa, perdiendo en ocasiones la objetividad de la elección del candidato ideal para el puesto vacante que debería estar sujeto a las competencias del ser, saber hacer y hacer.

Palabras Clave: Meritocracia, Sostenibilidad, Organizaciones, Sociedades, Sensibilidad

* Profesional en Comercio Internacional, Especialista en Gerencia de Negocios Internacionales - Especialista en Gerencia de Mercadeo en la Universidad Jorge Tadeo Lozano, Bogotá Colombia. Master Oficial en Educación y TIC (e-lerning), Universidad Oberta de Cataluya - UOC Barcelona España. Docente Asociado, Facultad de Estudios en Ambientes Virtuales- Programa de Negocios Internacionales- Universidad EAN-Colombia. Correo: monica.pastranag@ean.edu.co

** Profesional en Administración de Empresas de la Universidad EAN - Colombia. Coordinador Núcleo de Formación en Organizaciones y Estrategia. Docente de Herramientas de Gestión para el Siglo XXI, en la Universidad EAN - Colombia. Correo: fprieto.d@ean.edu.co 


\title{
THE PERCEPTION OF MERITOCRACY IN THE SELECTION PROCESS OF COLOMBIAN COMPANIES
}

\begin{abstract}
:
The concept of meritocracy can be related to a country's society and its form of government as for many people in order for meritocracy to exist democracy should prevail in a political system, otherwise it would be called dictatorship. It is for this reason that there is a need to investigate Colombian companies' personnel selection and evaluation process based on meritocracy. The present study is based on a descriptive approach applied to employees' at all organizational levels, including but not limited to strategic managers and tactical level positions, of companies located in the geographical area of Bogotá D.C. The data collection instrument used in the investigation was a closed question survey and its main objective was to understand employees' perception with regard to the meritrocratic criteria in the personnel selection and evaluation process.

Overall research findings show that the main variables in the personnel selection and evaluation process of the companies located in Bogotá are determined by personal references, work experience and academic studies; few companies have a recruitment and personnel selection handbook; in most cases the final selection decision is made by the company's Human Development Manager, thus losing objectivity, which should be based on the candidates' competencies such as: knowing to be, knowing to do, and knowing to know.
\end{abstract}

Keywords: Meritocracy, Sustainability, Organizations, Societies, Sensitivity

\section{A PERCEPÇÃO DA MERITOCRACIA NO PROCESSO DE RECRUTAMENTO NAS EMPRESAS COLOMBIANAS}

\section{Resumo:}

O conceito de meritocracia esta relacionado com os tipos de sociedades e formas de governo de um país, já que para muitas pessoas para que exista a meritocracia deve prevalecer à democracia em um sistema político, por que caso contrário seria uma ditadura, e é por esta razão que considerámos necessário investigar como está o estado atual da seleção e avaliação de pessoal das empresas colombianas baseado na meritocracia. Neste documento se evidencia os resultados de uma pesquisa descritiva, aplicado aos funcionários de todos os níveis organizacionais, incluindo, mas não exclusivamente para os cargos de gerência em nível estratégico e tático das empresas localizadas na zona geográfica de Bogotá D.C.

O instrumento de recolha de dados foi um questionário com perguntas fechadas, cujo principal objetivo era compreender a percepção dos trabalhadores a respeito dos critérios da avaliação meritocrática no processo de seleção e avaliação do pessoal. Os resultados generais da investigação demostram que as variáveis que predominam no processo de seleção do pessoal na maioria das empresas em Bogotá D.C. são determinadas por referências pessoais, experiência de trabalho e nível de estudos acadêmicos; poucas empresas possuem um manual de recrutamento e seleção de pessoal, e na maioria dos casos, a decisão final é determinada pelo chefe do departamento de desenvolvimento humano ou gerente da empresa, perdendo, às vezes, a objetividade da escolha do candidato ideal para o cargo, que deveria estar sujeita a competência do ser, saber fazer e fazer.

Palavras-Chave: Meritocracia, Sustentabilidade, Organizações, Sociedades, Sensibilidade. 


\section{INTRODUCCIÓN:}

Hoy en día la palabra meritocracia, presenta en la sociedad diferentes interpretaciones, tales como: poder, riqueza y/o posición social, pero este concepto en el proceso de selección y valoración del personal en una organización todavía no es clara su aplicación, surgen diferentes comentarios que entran a deducir que los procesos de meritocracia responden a criterios personales ya que no existe estándares de calidad predefinidos y, de ser así que muchos de estos procesos no son los más adecuados para administrar el personal 0 bien se establecen como un requisito que no necesariamente responde a la forma como se construyen las organizaciones para poder a llegar a ser más competitivo en el mercado, por lo anterior se consideró prioritario analizar qué tan inclinadas están las empresas en Colombia y sus empleados hacia el concepto y aplicación de la meritocracia dentro de sus procesos de selección y valoración del personal.

El análisis de la investigación fue tipo descriptivo, ya que fundamentalmente se está caracterizando una situación que es el de conocer la percepción que tienen los empleados y directivos respecto a los criterios de evaluación de los procesos meritocráticos en el proceso de selección y valoración del personal, investigación dirigida a las empresas del sector financiero de Bogotá D.C, arrojando como resultado sugerir acciones que permitan generar políticas y/o programas para fortalecer el proceso y valoración del personal acorde a las competencias del perfil del candidato y del puesto por ocupar.

\section{MARCO TEÓRICO:}

Dado que este artículo se centra en el concepto de la meritocracia y sus implicaciones en el proceso de selección y valoración del personal dentro de las organizaciones colombianas, resulta fundamental dar cuenta de la definición que ciertos autores les brindan al concepto.

La palabra merito se deriva del latín mereus, meritum, que significa con razón, con justicia, dignamente o digno de alabanza (Macionis y Plummer, 2007) en la cual su principal objetivo es recompensar al trabajador a merecer el cargo laboral que cumpla con sus competencias profesionales, laborales y emocionales en relación a otros candidatos que se encuentren también postulando.
Tiempos atrás, Young, brindo al término de meritocracia un sentido sarcástico y peyorativo que daba a entender que la meritocracia se termina convirtiendo en aristocracia arrogante, queriendo expresar el autor que las personas que contaban con buenas condiciones sociales era acreedores de ser premiados y respetados, con el paso del tiempo el término meritocracia empezó a tener un significado totalmente opuesto al original definido por Michael Young, dónde las personas tienen las mismas oportunidades para ser reconocidos en la sociedad y su retribución se puede expresar con dinero, ascensos de cargos laborales acorde a los méritos que haya hecho.

A lo largo de la investigación se validará la afirmación de Walzer (1997) que se resume en que los funcionarios del servicio imperial eran elegidos según una estricta aplicación de exámenes para garantizar al mejor candidato de entre aquellos que calificaban según requerimientos previamente definidos, pretendiendo el autor con esta premisa, que un cargo laboral vacante será ocupado por la persona que cumpla de forma satisfactoria con todos los requisitos definidos por la organización y que sobresalgan sus competencias de otras personas que se encuentre postulando para el mismo cargo.

Recopilando los sucesos más destacados de la meritocracia en Colombia se pueden referenciar los siguientes hechos:

* En 1938, se implantó la expedición de la ley 165 , bajo el mandato de López Pumarejo, que consistió en dar un giro político constitucional dirigido a cambiar las bases y organización del Estado y su administración.

* 1987, se expide la ley 61, que recupera el establecimiento del ingreso a la carrera administrativa por el sistema de mérito, sin embargo también permitió la inscripción automática para aquellos empleados que tenían antigüedad y se limitó a nivel nacional.

* En la constitución de 1991, se formalizó el principio de "ingreso por mérito" pero esta fue limitada y pospuesta debido a la resistencia que genera este mecanismo, en un medio acostumbrado a prácticas patrimonialistas o de botín político en el manejo de los cargos públicos. (Álvarez 2004). 
Hoy en día es difícil afirmar que la meritocracia en Colombia se ha usado un criterio transparente para la elección de sus funcionarios públicos ya que existe muchas razones para ser vulnerable por los mismos gobernantes y creadores de las leyes ya sea por su vinculación directa con el poder en la toma de decisiones o por la misma connotación que se brinda a la palabra democracia en las organizaciones 0 por la burocracia que predomina actualmente en el sistema social actual del país.

También han existido por parte de diferentes autores, críticas sobre la meritocracia, entre ellas se destaca la de Cociña (2013) dónde afirma: que una sociedad organizada en torno a la meritocracia corre el riesgo de erosionar las bases democráticas de la convivencia. La democracia es la organización de las decisiones colectivas en torno al principio de igualdad entre todos los participantes, por tanto participan tanto los talentosos como los más desaventajados. Una democracia fuerte no puede ser, por definición, sólo un gobierno de los mejores, sería en resumen, sólo una burocracia. El autor con esta afirmación, expresa que dentro del proceso de meritocracia dónde prevalece la libertad de expresión por parte del pueblo para elegir a sus representantes pueden filtrase personas no talentosas para ocupar un puesto vacante que en ocasiones son promovidas no por el cumplimiento de sus competencias laborales sino por disponer de referencias personales con poder en la organización que afectan el proceso trasparente de la meritocracia en la selección y valoración del personal tanto de un organización como de un país.

Para el gobierno el criterio de la meritocracia es difícil de mantener, debido a que existe la tendencia a gobernar autoritariamente; Por lo tanto, para Ramírez (2011) hay que poner límites a la democracia, es decir, que todo el poder debe estar sujeto a un control, con la intención de proteger a los ciudadanos en sus derechos civiles y políticos, frenar los abusos y que en la rotación en los puestos de poder se evite las preferencias y el gobierno injusto, con el objetivo de hacer una evaluación del desempeño de quienes ejercen el poder, sometiéndose a una rendición de cuentas.

\section{METODOLOGÍA:}

El alcance de la investigación es tipo descriptivo ya que el objetivo central es conocer la percepción de los empleados respecto a los criterios de evaluación de la meritocracia en el proceso de selección y valoración del personal, que de acuerdo a Sabino (1986), la investigación descriptiva, trabaja sobre realidades de hechos, y su característica fundamental es la de presentar una interpretación correcta. Para la investigación descriptiva, su preocupación primordial radica en descubrir algunas características fundamentales de conjuntos homogéneos de fenómenos, utilizando criterios sistemáticos que permitan poner de manifiesto su estructura o comportamiento. De esta forma se pueden obtener las notas que caracterizan a la realidad estudiada". (Pág. 51)

El instrumento de recolección de información, está constituido por una encuesta originalmente en idioma español, consta de 5 preguntas tipo cerrado, dónde se indagan de forma general sobre los elementos que son la base para analizar la sensibilidad de la meritrocracia en las empresas en Colombia, tales como: La opinión que tienen los empleados sobre la meritocracia, analizar las habilidades que consideran los encuestados imprescindibles 0 necesarias para lograr un buen desempeño en una empresa, explora cuáles son los aspectos que deben considerarse en un aspirante durante un proceso de selección de personal, reconocer cuáles son las etapas del proceso de selección que atravesó el encuestado para ingresar a la compañía, identifica las razones por las cuales se discriminan las personas en organización y que obstaculizan su ascenso y/o escalafón.

Las preguntas fueron diseñados basados en el modelo de comportamiento planificado TPB (Theory of Planned Behavior), dónde se abordaron los tres tipos de creencias que permiten conocer el comportamiento de un individuo actitudes, normas subjetivas y control comportamental percibido-, aplicadas específicamente a la intención de los empleados encuestados por el tema de la sensibilidad de la Meritrocracia en las pymes en Colombia.

La recolección de información se realizó en Bogotá, de forma física no virtual para validar que la persona objetivo central del problema de investigación fuera quién realmente la presentará.

La población es finita, se delimito una muestra representativa en relación a un sector, que para este caso fue el de servicios - entidades financieras, arrojando un total de 250 encuestas.

El resultado del instrumento de recolección de información fue satisfactorio, concluyendo el ejercicio con la tabulación de datos y el diseño de gráficas por cada pregunta definida en el cuestionario.

La selección de la muestra para el desarrollo de la investigación fue representativa para validar apreciaciones sobre el tema del proyecto que es la LA PERCEPCIÓN DE LA MERITOCRACIA EN EL PROCESO DE LA SELECCIÓN DE PERSONAL EN LAS EMPRESAS COLOMBIANAS. 


\section{RESULTADOS Y DISCUSIÓN:}

A continuación se presentan los resultados obtenidos con el proceso de recolección de información expresados con un análisis cuantitativo y cualitativo, dónde se permite distinguir las principales percepciones que tienen los empleados del sector financiero en Colombia respecto al proceso de la meritocracia para la selección y valoración del personal.
Entre un $60 \%$ y el $80 \%$ de las personas encuestadas consideran que nunca 0 que no saben si hay discriminación en sus empresas, parece ser que muestran una indiferencia relacionada con el tema o ignoran por completo lo que sucede en lo referente al asunto al interior de las organizaciones para las cuales trabajan.

Figura No 1

Aspectos que se consideran claves para discriminar las personas en la Organización

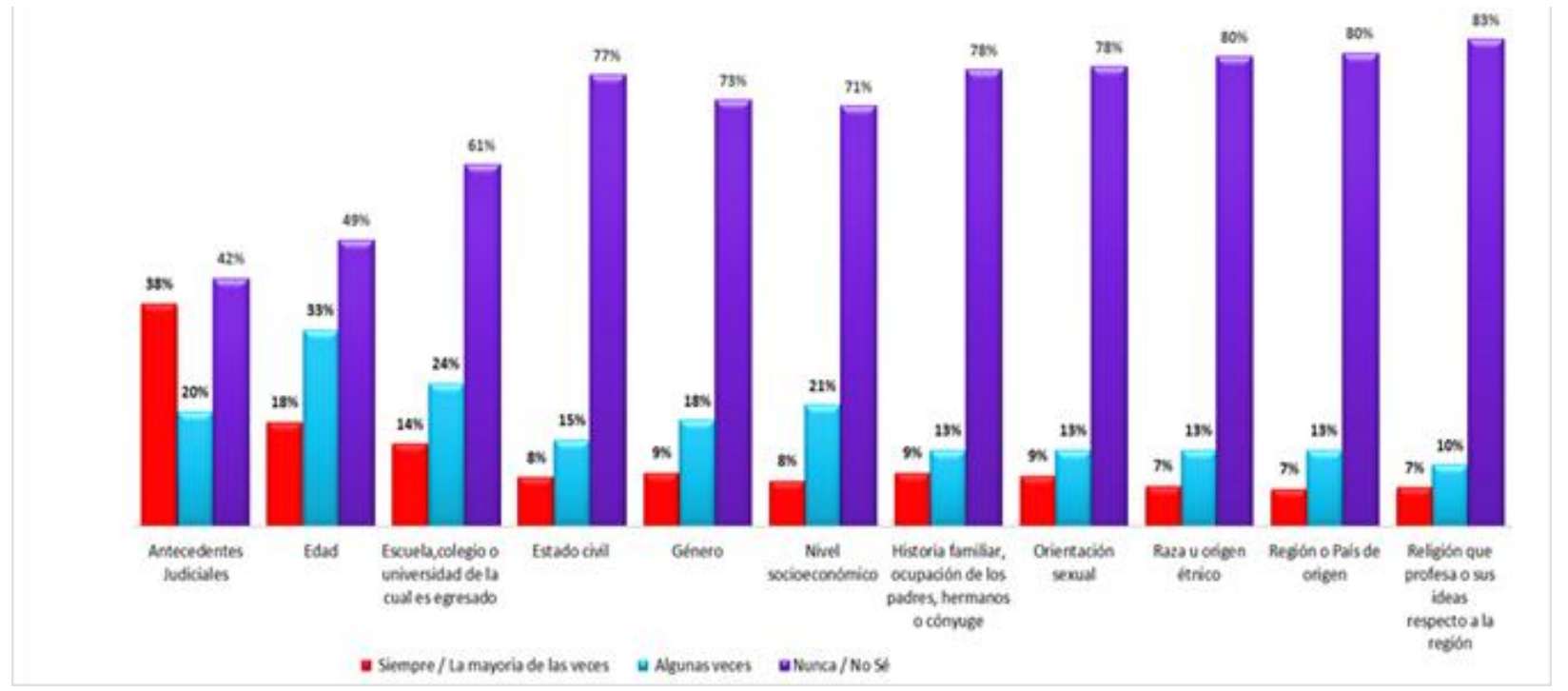

Fuente: Pastrana G. y Prieto P. (2015)

En la figura No 1 , se evidencia que un $58 \%$ de los encuestados consideró, que en las empresas en donde trabajan, se discrimina a las personas por tener antecedentes judiciales, el $51 \%$ opinó que hay discriminación por la edad. En Colombia, los mayores de 40 años se sienten discriminados por la edad. Mientras tanto, los jóvenes entre 18 y 24 años dicen ser rechazados laboralmente por la falta de experiencia debido a su edad, cerca del $40 \%$ consideró que existe discriminación relacionada a la institución educativa de la cual egresaron lo cual está ligado al nivel socioeconómico que en la gráfica se muestra cercano al $30 \%$.
En la figura No 1, se evidencia que un $58 \%$ de los encuestados consideró, que en las empresas en donde trabajan, se discrimina a las personas por tener antecedentes judiciales, el $51 \%$ opinó que hay discriminación por la edad. En Colombia, los mayores de 40 años se sienten discriminados por la edad. Mientras tanto, los jóvenes entre 18 y 24 años dicen ser rechazados laboralmente por la falta de experiencia debido a su edad, cerca del 40\% consideró que existe discriminación relacionada a la institución educativa de la cual egresaron lo cual está ligado al nivel socioeconómico que en la gráfica se muestra cercano al $30 \%$. 
Entre un $60 \%$ y el $80 \%$ de las personas encuestadas consideran que nunca 0 que no saben si hay discriminación en sus empresas, parece ser que muestran una indiferencia relacionada con el tema o ignoran por completo lo que sucede en lo referente al asunto al interior de las organizaciones para las cuales trabajan. De manera general la figura No 2 refleja que en promedio un $75 \%$ de las personas encuestadas consideran que "siempre o la mayoría de las veces", o "algunas veces" existen personas ejercen una poderosa influencia sobre los demás.
Vale la pena resaltar que en el ítem de esta pregunta, en donde se indaga si el ambiente de trabajo en un área 0 grupo depende de las decisiones 0 actitudes del jefe, la mayoría de las personas, representadas en un $82 \%$ contestaron afirmativamente, lo que probablemente podria obedecer a dificultades de liderazgo y manejo de grupos por parte de los jefes encargados, lo anterior generaría una atmosfera inapropiada para el trabajo. Este tipo de situaciones a corto plazo, se podría reflejar en deserción de personal, afectando así la operación de la empresa.

Figura № 2

Sucesos que prevalecen en la organización para la

toma de decisiones

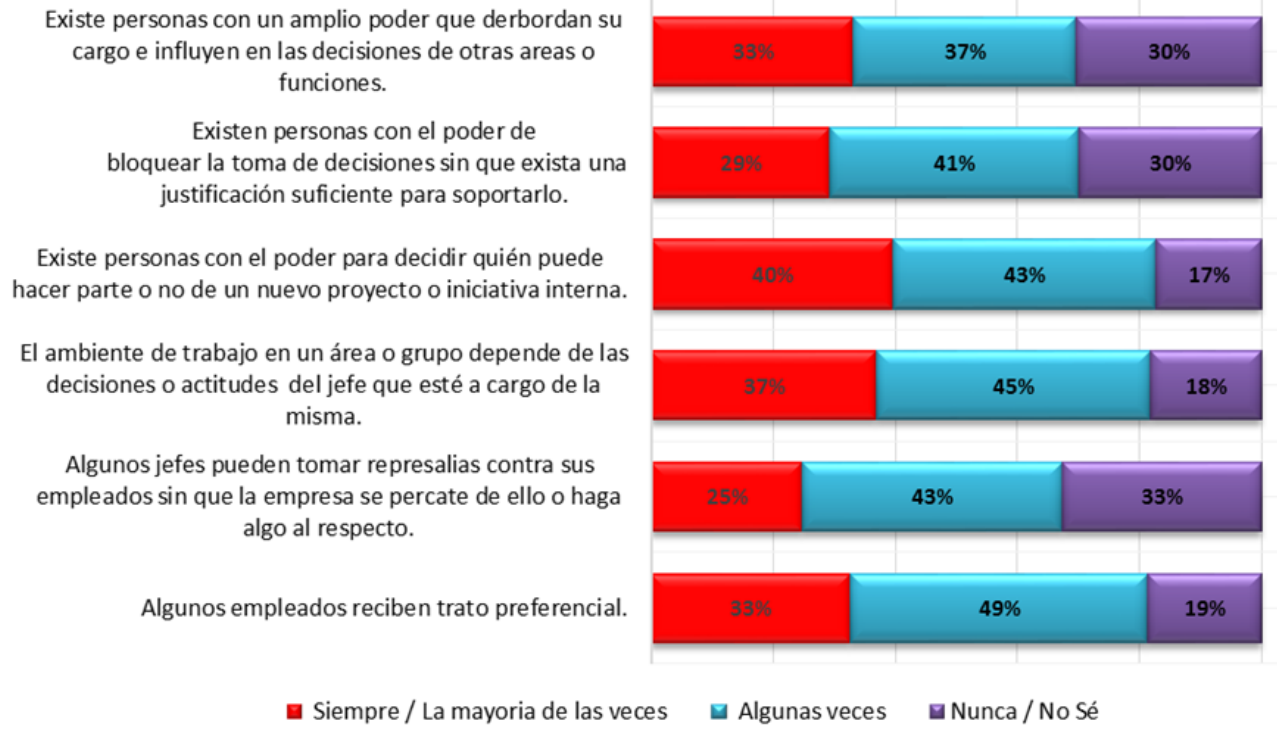

Fuente: Pastrana G. y Prieto P. (2015)

El $82 \%$ de las personas encuestadas, dado de la sumatoria de "siempre o la mayoría de las veces" y "algunas veces", consideran que hay empleados que reciben trato preferencial, lo que podría llegar a afectar directamente la convivencia, las relaciones entre el personal y en general el ambiente de trabajo.

También se podría presentar que debido al trato preferencial que algunos empleados reciben obtengan beneficios que harán que se genere desigualdad entre ellos, teniendo en cuenta que posiblemente se encuentren capacitados al mismo nivel.
Cerca de un $70 \%$ de las personas encuestadas contestó de manera afirmativa al siguiente ítem, "algunos jefes pueden tomar represalias con sus empleados sin que la empresa se percate de ello o haga algo al respecto". Existe la creencia de que los jefes, de manera general en las empresas, actúen de esta manera, sin embargo no solo se observa este comportamiento sino también la infracción que probablemente algunos jefes pudieran estar cometiendo al reglamento interno de trabajo; lo que podría llamarse acoso laboral, por tomar represalias sin que la organización se percate de estas 0 se haga algo al respecto, como lo dice el enunciado. 
Figura No 3

Percepción del proceso de selección de personal en

la empresa que labora actualmente

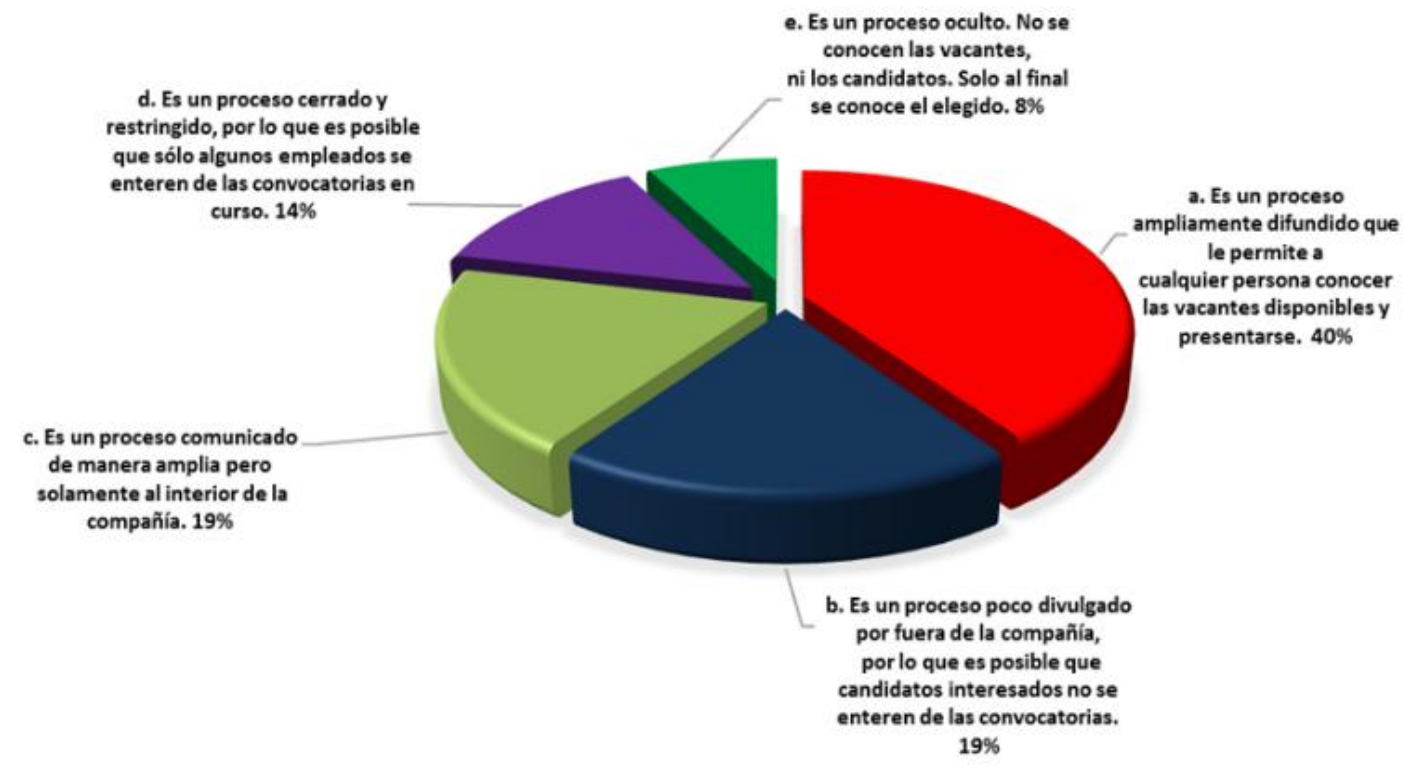

Fuente: Pastrana G. y Prieto P. (2015)

Según el autor, Chiavenato (2000), se establece que: "EI proceso de selección no es un fin en sí mismo, es un medio para que la organización logre sus objetivos". El proceso de selección consiste en una serie de fases iniciales que deben ser claramente definidas, por lo anterior de acuerdo a la figura No 3 , el $40 \%$ de las personas encuestadas consideraron que los procesos de selección en sus empresas son ampliamente difundidos y que las personas se enteran de las vacantes disponibles, lo que podría demostrar que las empresas en donde laboran estas personas tienen claridad de cómo administrar adecuadamente el recurso humano. Al ser un proceso ampliamente difundido incluye al personal interno y a las personas externas de la organización.
También podemos analizar en los resultados representados en la figura No 3 , que en porcentajes iguales, $19 \%$, algunos piensan que el proceso es poco divulgado por fuera de la compañía, mientras que el otro $19 \%$ opinó que el proceso se divulga ampliamente pero solo dentro de la compañía. El $14 \%$ de las personas encuestadas consideró que el proceso de convocatoria, dentro de la empresa, es un proceso cerrado y restringido y que solo algunos empleados se enteren. Solo un pequeño números de encuestados representados en un $8 \%$ manifestó que los procesos de selección en sus empresas se hacen de manera oculta. 
Figura No 4

Percepción del rol desempeñado por el Jefe inmediato.

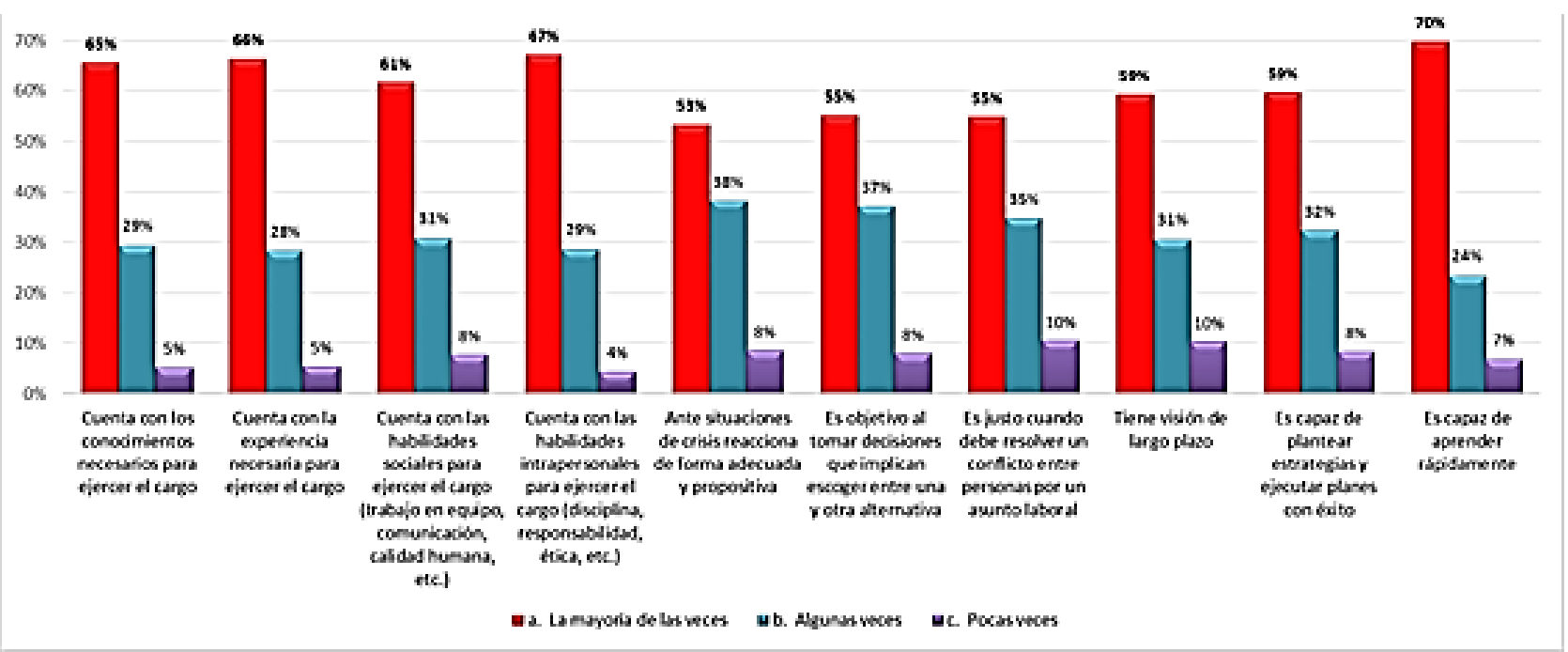

Fuente: Pastrana G. y Prieto P. (2015)

En esta pregunta la mayoría de personas que participaron en la encuesta respondieron que su jefe inmediato cumple con ciertos aspectos y responsabilidades que deben tener para asumir el cargo asignado.

a) Cuenta con los conocimientos necesarios para ejercer el cargo. La respuesta con mayor porcentaje obtenido fue a. La mayoría de las veces con un porcentaje del $65,44 \%$. Este resultado nos indica que un buen segmento de la muestra refleja que el jefe si cuenta con los conocimientos para ejercer el cargo asignado; no obstante la muestra refleja un porcentaje del $35 \%$ bastante alto, que califica al jefe que no tiene los conocimientos necesarios para ejercer el cargo como lo indica el $29,46 \%$ al contestar algunas veces y el $5,10 \%$ pocas veces. Lo cual es un resultado preocupante, ya que el $35 \%$ de los encuestados manifestaron que el jefe no tienen el conocimiento suficiente para el desempeño del cargo, esto nos quiere decir que el jefe no los puede apoyar u orientar en caso dado a la solución de un problema que se le presente al subalterno. igualmente mostraría que no tiene autoridad de conocimiento para presentar una corrección adecuada. b) Cuenta con la experiencia necesaria para ejercer el cargo. Los resultados son muy parecidas al punto anterior, donde la respuesta $a$. La mayoría de las veces cuenta con un porcentaje del $66,29 \%$ de los encuestados. Pero esta vez nos indica que un buen segmento de la muestra refleja que el jefe si cuenta con la experiencia necesaria para ejercer el cargo asignado. El $34 \%$ restante como ya sabes que es bastante alto, donde los encuestadores califica al jefe que no tiene la experiencia necesaria para ejercer el cargo, como lo indica el 28,33\% al contestar algunas veces y el $5,38 \%$ pocas veces. Tomado estos resultados nos quieren decir que muchos jefes conocen los cargos lo cual se manifiestan en experiencia y eso es favorable a la entidad como tal y el resultado inferior del $34 \%$ nos indica que los jefes son recién egresados de las instituciones de enseñanza superior y los ponen a dirigir tan solo con el conocimiento obtenido en la aulas de clase. 
c) Cuenta con las habilidades sociales para ejercer el cargo (trabajo en equipo, comunicación, calidad humana, etc.). Los resultados obtenidos en este punto muestran que el $61,47 \%$ de los encuestados dicen que su jefe la mayoría de veces cuenta con habilidades sociales para ejercer el cargo. Un $30,88 \%$ de los encuestados opinan que algunas veces y el $7,65 \%$ pocas veces su jefe tiene las habilidades sociales para ejercer sus funciones. El resultado de la encuesta nos refleja que el $61 \%$ de los jefes tiene empatía con los subalternos, esto quiere decir que se trabaja armónicamente dentro de las actividades desarrolladas en la empresa; mostrando así un $30 \%$ que no encuentra empatía con el jefe no realizándose un trabajo ameno y agradable dentro de la misma.

d) Cuenta con las habilidades intrapersonales para ejercer el cargo (disciplina, responsabilidad y ética) observando los dados recogidos tenemos que el $67.14 \%$ de las personas encuestas respondieron la a. La mayoría de las veces su jefe cuenta con las habilidades interpersonales. Un $28.61 \%$ porciento contestaron b. Algunas veces y el $4.25 \%$ porciento pocas veces. Según $28.61 \%$ de las personas encuestadas sus jefe no manejas una buena convivencia a dentro del ámbito laboras y nos preguntamos ¿por qué marcaron a opción de algunas veces?, Será que hay indisposición y así el jefe de los $4.25 \%$ encuestados respondiendo pocas veces.

e) Ante situaciones de crisis reacciona de forma adecuada y propositiva. En esta punto las personas encuestadas tan solo el $53.26 \%$ lo cual es preocupante ya que se refleja la mitad de los jefes no reaccionas de forma adecuada frente a la solución de problemas lo cual puede afectar a institución por malas decisiones, la otra mitad estaríamos hablando del $38.24 \%$ que respondieron algunas veces y el $8.50 \%$ con muy pocas veces.

f) Es objetivo al tomar decisiones que implican escoger entre una y otra alternativa. Verdaderamente los jefes de las personas encuestadas o por lo menos gran parte de la muestra correspondiendo al $37.11 \%$ respondieron algunas veces y el $7.93 \%$ pocas veces, postrándonos así que ellos no toma decisiones por el bien de la empresa sino al parecer de ellos diciendo entre los dientes "yo se mas que ustedes y no tienes derecho a opinar". g) Es justo cuando debe resolver un conflicto entre personas por un asunto laboral. En este tipo de situaciones ¿será que el jefe tiene preferencia con algunos empleados?, pues el $34.84 \%$ de las personas encuestadas respondieron algunas veces y el $10 \%$ respondió pocas veces, cifra de importancia ya que no se debería presentar este tipo de preferencias sino reconocer los esfuerzos de todos sus empleados ya que todos trabajan para él y ellos poder ganas su sueldo sin ningún problema, recordando que cada persona tiene sus derechos y obligaciones que deben hacerse cumplir por el jefe. Pero pues en todo caso el $54.67 \%$ respondieron la mayoría de veces.

h) Tiene visión de largo plazo. Respecto a la visión tengamos en cuenta primo que es, es tener metas y que se haga todos lo necesaria para que se haga realidad. Lograr metas no solo es felicidad del jefe sino también de sus empleados. Los resultados que obtuvimos en la encuestas el $59.21 \%$ los empleados tuvieron como opción la mayoría de veces queriendo decir esto que sus jefes si cumple con la visión que se propone la empresa. De todas formas la cifras de personas contestaron algunas veces que fue el $30.59 \%$ y los que dijeron pocas veces el jefe no cumple con los logros propuesto fue el $10 \%$ de la muestra.

i) Es capaz de plantear estrategias y ejecutar planes con éxito. Normalmente el jefe o gerente debe tener la capacidad de crear estrategia y aplicarla pero pues la encuesta realizada nos muestra que el $59.49 \%$ claro esta una buena parte de la muestra respondieron la mayoría de veces. Pero en todo caso es preocupante la cifra de del $32.29 \%$ contestaron algunas veces y el $8.22 \%$ pocas veces. ¿ que pasara con los gerentes de hoy en día que no tienen creatividad para plantear estrategias y ejecutarlas?.

j) Es capaz de aprender rápidamente. al parecer se obtuvo un $70 \%$ de los encuestados que el jefe la mayoría de veces aprende rápidamente este porcentaje es bueno ya que eso es muy importante para la empresa para poder estar actualizada. De todos modos queda un $24 \%$ con algunas veces y $7 \%$ pocas veces pues estas empresas mientas sus jefes no aprendan rápido el nuevo mercado se tendría un retraso y eso no es bueno para estas nuevas generaciones de gerencia que se vienen creando. 
Figura No 5

Percepción del rol desempeñado por sí mismo

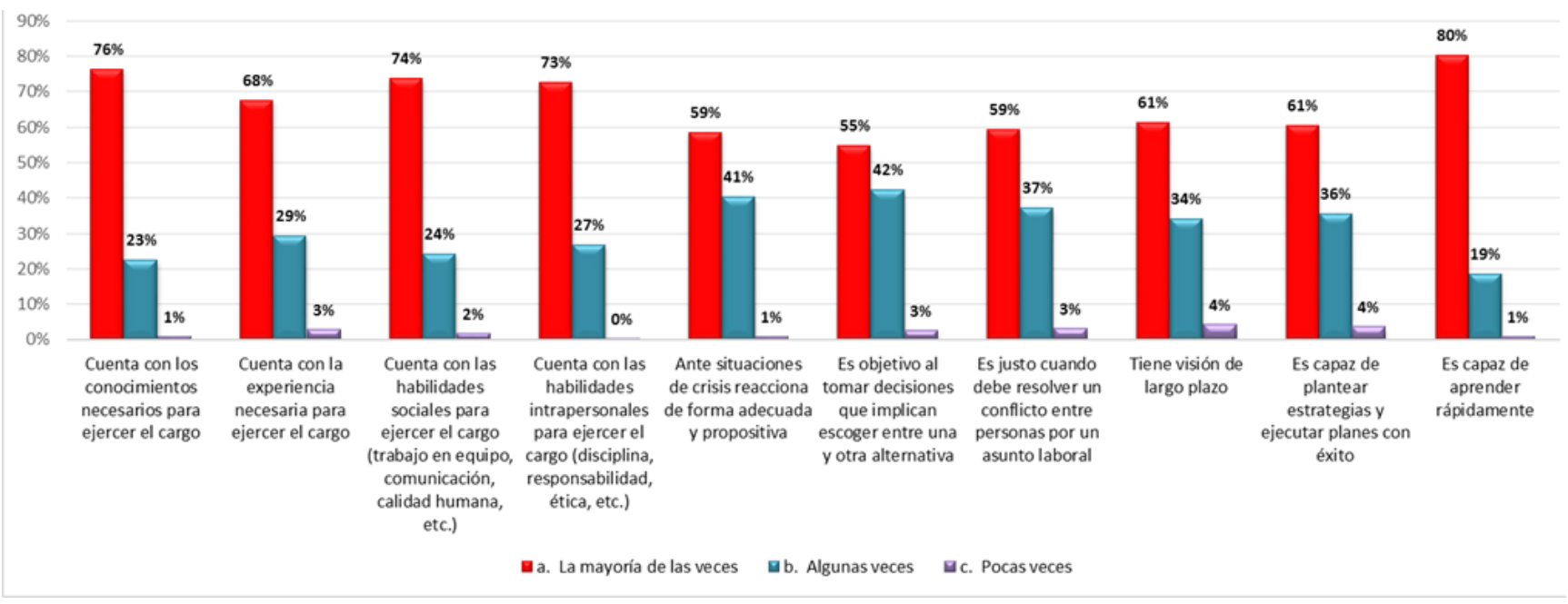

Fuente: Pastrana G. y Prieto P. (2015)

En este punto los encuestadores se miran a sí mismos y nos dicen como son ellos en las distintas situaciones que se plantean y donde se pueden observar que ellos mismos se dan un porcentaje más alto que cuando calificaron a su jefe y sus compañeros

a) Cuenta con los conocimientos necesarios para ejercer el cargo: El $76 \%$ de la muestra dicen que la mayoría de veces entonces, ellos tal vez tiene su cartón de grado, pregrado, posgrado y demás estudios requeridos para ocupar su cargo, pero un suma entre los que respondieron algunas veces y pocas veces corresponde a un $24 \%$ y ellos solo tendrán el grado escolar y tu técnico o tecnólogo y tiene un cargo que no corresponde a sus estudios. ¿TIENEN PALANCA?

b) Cuenta con la experiencia necesaria para ejercer el cargo: un $33 \%$ porcientos de los encuestados responden entre algunas y pocas veces cuentan con la experiencias necesaria, estas personas serán egresados de los estudios superiores y entran la campo laboral o que nunca habían trabajado en ese cargo por ende no tienen la experiencia necesaria. $Y$ viéndolo bien no es una cifra muy alta pues ya que el $68 \%$ de ellos si cuentan con la experiencia, es decir que llevan más de 2 años trabajando en el mismo campo de acción. c) Cuenta con las habilidades sociales para ejercer el cargo (trabajo en equipo, comunicación y calidad humana, Si juntamos a las personas que respondieron algunas y pocas veces nos da un porcentaje del $25 \%$ lo cual se puede interpretar de esto ellos no cuentan con las habilidades sociales requeridas, pues esto quiere decir que no tiene buena comunicación y otras serie de habilidades los cual es necesario para la mayoría de trabajos en los cuales hay trabajos en equipo y si no se tiene esto pues no habrá un buen ambiente de trabajo. El $74 \%$ porcentaje alto lo cual es bueno, ya que el manejo de una empresa requiere de buenas relaciones sociales entre cada uno de los miembros.

d) Cuenta con las habilidades intrapersonales para ejercer el cargo (disciplina, responsabilidad y ética) encontramos algo muy favorable tenemos un $0 \%$ en la respuesta de pocas veces los cual nos dice que el $100 \%$ tiene entre algunas veces y la mayoría de veces, esto es positivo ya que en el sitio de trabajo hay un buen ambiente laboral y cada quien cumple con sus deberes como profesional. Si se mantiene podríamos tener excelentes resultados dentro de empresas. 
e) Ante situaciones de crisis reacciona de forma adecuada y propositiva. La respuesta con mayor porcentaje fue la mayoría de veces que corresponde al 59\%, estas personas tienen la capacidad de reaccionar adecuadamente ante situaciones de crisis. Esos es bueno dentro de una empresa y tengan personas con esta capacidad de resolver problemas y saber que un poco más de la mitad de la muestra tiene esta capacidad. Por otro lado el $42 \%$ contestaron algunas veces y pocas veces lo cual no es bueno pero una empresa requiere de personas con adecuadas reacciones frente a a una situación de crisis.

f) Es objetivo al tomar decisiones que implican escoger entre una y otra alternativa. Sumando los resultados de la mayoría de veces y algunas veces corresponde al $98 \%$ cifra alta, donde podríamos decir que estas personas tiene claro que una toma de decisiones es importante ya que si se toma mal una decisión puede traer fuertes consecuencias a la empresa. Y tan solo el $3 \%$ no son seguros al tomar una decisión.

g) Es justo cuando debe resolver un conflicto entre personas por un asunto laboral. Tengamos claro que ser justo es el camino correcto para resolver problemas, en nuestra encueta encontramos una buena cifra donde los encuestados dicen que ellos la mayoría de veces y algunas veces son justo, este porcentaje es del $98 \%$, lo cual es excelente para una buena convivencia dentro de los campos laborales e importante que cada empleado se justo al momento de resolver conflictos y no crear más problemas entre el mismo conflicto. $Y$ por último los un $3 \%$ digamos que no entra mucho en la solución de conflictos no les interesan y los evita a toda costa.

h) Tiene visión de largo plazo. La visión a largo plazo es importante dentro de una empresa y que los empleados tienes actitud positiva de que si se va a lograr esa meta propuesta. Un 39\% entre algunas veces y pocas veces no son seguros de que la empresa pueda conseguir esas metas propuestas aun haciendo todo los esfuerzos posibles para lograrlo, pero tenemos un porcentaje alto donde la mayoría de veces son positivos ante las visiones de la empresa este es de un $61 \%$ de la muestra. i) Es capaz de plantear estrategias y ejecutar planes con éxito. El planteamiento de estrategias y ejecutarlas es una de las capacidades que todo empleado debería tener. En nuestras encuestas un $60 \%$ dice tener la capacidad la mayoría de veces lo cual es un porcentaje alto ya que es importante tener funcionarios dentro de las empresas con esas capacidades. Lo preocupante es el $40 \%$ de ellos que algunas veces y pocas veces no requieren de esta capacidad, lo cual la empresa podría tener problemas con estas personas ya que se necesita esta habilidad para que la empresa puede tener buenas estrategia ya sea de venta, solución de problemas, desarrollo de actividades, etc... dentro de la misma.

j) Es capaz de aprender rápidamente. Como se puede observar es el porcentaje más alto de nuestro análisis donde los encuestados dicen tener la mayoría de veces un aprendizaje rápido, podemos deducir de ello que son jóvenes entre los 20 y 30 años de edad. Los que respondieron algunas veces son el $19 \%$ y el $1 \%$ tal vez ellos no tengan la misma rapidez que tiene los otros pero no es un porcentaje que sea significativo.

\section{CONCLUSIONES:}

La percepción de la mayoría de encuestados en relación al uso efectivo de la meritocracia en el proceso de selección y valoración de personal arroja que las empresas en donde trabajan actualmente, se discrimina a las personas por sus antecedentes judiciales o también por su años dejando en ocasiones a un lado la experiencia laboral, también se destaca que existen personas en la organización que ejercen poderosa influencia en la selección del personal y por su poder frente a las decisiones en ocasiones no se llega a cumplir con los procesos establecidos por la organización para la contratación del personal.

También se destaca que un buen porcentaje de los encuestados afirma que su jefe inmediato cuenta con los conocimientos para ejercer el cargo asignado; no obstante muestra desacuerdo con el cargo que se encuentra ejerciendo actualmente ya que no corresponde con sus estudios, experiencia laboral y competencias socio humanísticas. 
Mónica Alejandra Pastrana Granados - Franklin Ariel Prieto Pinto

En general el público no tiene tan claro el concepto de meritocracia en donde se establece que el talento es el criterio para definir los ascensos y aumentos de salario, por otro lado también relacionan la formación académica y las capacidades técnicas para ejercer una labor, en general los encuestados perciben el concepto de meritocracia en relación a la selección del personal con las capacidades y la formación académica.

Como recomendación general, para que las empresas en Colombia exista una percepción homogénea del concepto de meritocracia para la selección y valoración del personal, se debe disponer de evaluadores externos a la organización que puedan garantizar una evaluación objetiva del cumplimiento de los méritos del candidato en relación al perfil profesional que requiere la empresa del cargo vacante por ocupar, dónde no se encuentre influenciado por los intereses personales 0 sectoriales 0 que dado caso sean afectados por exclusiones sexistas, racistas, propios y/o predominantes de nuestro país.

Por lo anterior, es necesario empezar aceptar que la meritrocracia en nuestro país aún no existe y más que un procedimiento o proceso formal que una empresa debería siempre aplicar y este proceso podría ser efectivo, si el país fuera justo en su toma de decisiones y en el ofrecimiento de oportunidades y no estuviera cargado por el poder que tiene pocos y la distancia entre la democracia y el verdadero talento laboral, dónde no existirá grupos sectoriales, oligopolios empresariales si no se entrará a evaluar el desempeño de los trabajadores por competencias no solo del ser sino del saber hacer y del hacer.

\section{REFERENCIAS:}

Álvarez A. (2004). Los Sistemas de Administración de Personal en el Sector Público. ESAP, Bogotá D.C

Arrighi,G. (2001). Caos y orden en el sistema- mundo moderno. Ediciones Akal

Armas, Jorge (2008). Los falsarios de la historia "La realidad de la meritocracia" Recuperado el 7 de agosto de 2014 de http://www.revistalarazonhistorica.com/4-6/

Bodde,, Derke. "China: A Teaching Workbook". Columbia University.

Bolivar, A. (2004). Los patios interiores de la democracia: subjetividad y politica: Norbert lechner. Polis, revista universidad bolivariana, Vol. 2 (007), pp. 2-6 .Recuperado
http://www.plataformademocratica.org/Public acoes/14433_Cached.pdf

Centro Virtual de Cervantes. (1997-2014). Pragmática. [Versión Electrónica]. Madrid: Instituto Cervantes.,http://cvc.cervantes.es/ensenanz a/biblioteca_ele/diccio_ele/diccionario

/pragmatica.htm

Contreras, E. M. (jueves, 2 de septiembre de 2010). ideologias . Obtenido de http://historiaideologias.blogspot.com/2010/0 9/la-meritocracia.html?m=1

Cociña, M. (07 de 06 de 2013). CIPER, Centro de Investigación Periodística. Obtenido de http://ciperchile.cl/2013/06/07/cincoargumentos-contra-la-meritocracia/

Dapena, L. (2003). Núcleos de vida ciudadana: racionalidades y coyunturas. Recuperado del sitio de internet de la biblioteca Ciudades para un mundo más sostenible: http://habitat.aq.upm.es/nvc/aldap_5.html

Elman, S. (1968). Primitive Social Organization an evolutionary perspective. New York: Random House

Hamilton, D. (1994). Comments on Schaniel and Neale's Comments on Hamilton.

Hacker, J. S. y P. Pierson. 2011. Winner-Take-All Politics. New York. NY: Simon and Schuster.

Hayes, C. L. 2012. Twilight of the Elites. New York, NY: Crown

Helio, J. (1986). Raza, cultura y clase de la integración de las sociedades. Recuperado de http://www.jstor.org/discover/10.2307/23395 993?uid=3737808\&uid=2\&uid=4\&sid=21104 066345431

Hincapié, S. (2014). Sociedades, formas de gobierno y decisiones. Manuscrito no publicado, Facultad de Administración de Empresas, Universidad EAN, Colombia.

Jiménez B., William Guillermo. (2012). Teoría y práctica del sistema de mérito a través de las reformas del Estado en Colombia. Reflexión Política, Diciembre- Mayo, 142$157 . \quad$ Recuperado de http://www.redalyc.org/pdf/110/11025028011 .pdf 
Journal of Economic Issues, Vol. 28, pp. 891-894. Recuperado de http://www.jstor.org/stable/4226860

Kazin, Edwards, and Rothman (2010), 142. One of the oldest examples of a merit-based civil service system existed in the imperial bureaucracy of China.

Konner, Melvin (2003). Unsettled: an anthropology of the Jews. Viking Compass. p. 217. "China is the world's oldest meritocracy"

Lacoviello, M; Luciano, S. (2011). De partidocracias rígidas a meritocracias flexibles en América Latina, DAAPGE, año 11, № 16, 2011. Pp. 51-95.

Levinson, David; Sadovnik, Alan R. (2002). Education and Sociology: An Encyclopedia

Lewin, R. (2000). Complexity, Life at the edge of Chaos. Chicago: University Of Chicago Press.

Maturana, R., Humberto y Francisco Varela G. (2003). El árbol del conocimiento. Buenos Aires: Lumen.

Macionis, John y Plummer, Ken (2007). Sociología. Madrid: Pearson Prentice Hall.

Mijares, M. (2001). Formas de Gobierno. Recuperado de http://books.google.es/books? $\mathrm{hl}=e s \& \mid \mathrm{r}=\& \mathrm{id}=\mathrm{aij}-$ SIMz3VgC\&oi=fnd\&pg=PA7\&dq=formas + de +gobierno\&ots $=q G O a h 4-$ vBZ\&sig=mSDVCc6UcezGsUUhSaz42GkSa $Q A \# v=$ onepage $\& q=$ formas $\% 20 \mathrm{de} \% 20 \mathrm{gobi}$

Miriam Henry (1988). Understanding Schooling: An Introductory Sociology of Australian Education. Psychology Press. p. 81.

Mora, G. (2003). Racionalidad y tipos de racionalidad. Revista de la Filosofía de la Universidad de Costa Rica, 41(103), 93-100. Recuperado de

http://inif.ucr.ac.cr/recursos/docs/Revista\%20 de\%20Filosof\%C3\%ADa\%20UCR/Vol.\%20X Ll/No.\%20103/Racionalidad\%20y\%20tipos\% 20de\%20racionalidad.pdf
Muñoz Aravena, W. (2008). Cuando el mérito acentúa la desigualdad. Enfoques, (9), 247- 261. Recuperado de http://www.redalyc.org/pdf/960/96060910.pdf

North, D.C. (2000). La Evolución Histórica de las Formas de Gobierno. Revista de Economía Institutional, 2 (2), 113-148. Recuperado de http://www.scielo.org.co/scielo.php?pid=S01 24-59962000000100007\&script=sci_arttext

Pineda, M. (s.f.). El concepto de la racionalidad. Revista Actualidades Investigativas de la Educación .Recuperado de http://servicio.bc.uc.edu.ve/educacion/revista la1n2/1-2-4.pdf

Ramírez, C. A. (16 de 06 de 2011). ALAl, América Latina en Movimiento. Obtenido de http://alainet.org/active/47371\&lang=es

Sabino (1986), Metodología de la Investigación. Recuperado de: https://bianneygiraldo77. wordpress.com/cate gory/capitulo-iii/

Sartori, G. (1999). Elementos de teoría política. vol. (93). Madrid: Alianza Editorial. Recuperado de

http://scholar.google.es/scholar?hl=es\&q=de +monarqu $\%$ C3\%ADa+a++tirania

$\& b \operatorname{tn} G=\& \mid r=$

Schwarz, Bill. (1996). The expansion of England: race, ethnicity and cultural history. Psychology Pres. ISBN 0-415-06025-7.

Tan, Chung; Geng, Yinzheng (2005). India and China: twenty centuries of civilization interaction and vibrations. University of Michigan Press. p. 128. "China not only produced the world's first "bureaucracy", but also the world's first "meritocracy"'"

Tauranie, A. (s.f.). Los caminos sinuosos de la democracia. Recuperado de http://www.plataformademocratica.org/Public acoes/14434_Cached.pdf

Taylor \& Francis. p. 436. "most common definition of meritocracy conceptualizes merit in terms tested competency and ability, and most likely as measured by $I Q$ or standardized achievement tests" 
Terraza, A. (2006).Cultura, modos de vida y evolución humana: relaciones categoriales. Boletín De Antropología Americana, (42), 5-32. Recuperado de http://www.jstor.org/discover/10.2307/40978 384 ? uid $=3737808 \&$ uid $=2134 \&$ uid $=2 \&$ uid $=70$ \&uid=4\&sid=21104066435751

Trovero, J. (2012). Max Weber y el proceso de racionalización occidental: consideraciones en torno a su influjo sobre la tipología de la dominación legítima.Universidad Nacional de La Plata, La Plata, Argentina.

Tucker, Mary Evelyn (2009). "Touching the Depths of Things: Cultivating Nature in East Asia". Ecology and the environment: perspectives from the humanities (Harvard Divinity School): 51. "To staff these institutions, they created the oldest meritocracy in the world, in which government appointments were based on civil service examinations that drew on the values of the Confucian Classics"

Vidal, G. (2008).La teoría de la elección racional en las ciencias sociales. Revista Sociológica, 23(67), 221-236. Recuperado de ww.revistasociologica.com.mx/pdf/6709.pdf

Waleska, A. (2008). Cuando el mérito acentúa la desigualdad. Revista enfoques. Año VI, edición No 9. Pp. 247-261.

Walzer, Michael (2004). Las esferas de la justicia. Una defensa del pluralismo y la igualdad. México: Fondo de Cultura Económica.

Weber, M. (1956). Conceptos sociológicos fundamentales. En: Economía y Sociedad. México: Fondo de cultura Económica

Weber, M. (1975) "Economía y Sociedad". México: Fondo de Cultura Económica, Tomo I.

Weber, M. (2007). Sociología del poder: Los tipos de dominación. El Libro De Bolsillo - Bibliotecas De Autor - Biblioteca Weber. Madrid :Alianza.

Wright, E. O. 2013. "Transforming Capitalism through Real Utopias." American Sociological Review 78(1):1-25.
Wright, E. O. 2010. Envisioning Real Utopias. New York, NY: Verso Books.

Yao,X. (2001). Confusionismo. Ed Akal

Young, M. 1961. The Rise Of Meritocracy. Mitcham, Victoria: Pelican.

Young, Michael (1958). The rise of the meritocracy, 1870-2033: An essay on education and inequality. London: Thames \& Hudson. 